\title{
Diagnósticos de enfermagem de pacientes no período pré-operatório de cirurgia cardíaca*
}

\author{
NURSING DIAGNOSIS OF PATIENTS IN THE PREOPERATORY PERIOD OF CARDIAC SURGERY \\ DIAGNÓSTICOS DE ENFERMERÍA DE PACIENTES EN EL PERIODO PRE OPERATORIO \\ DE CIRURGÍA CARDIACA
}

\author{
Luzia Elaine Galdeano1, Lídia Aparecida Rossi², Termutes Michelin Pezzuto³
}

\begin{abstract}
RESUMO
Oobjetivo deste estudo foi identificar os diagnósticos de enfermagem de pacientes no periodo pré-operatório de cirurgia cardíaca. Para a coleta de dados foi elaborado e validado um instrumento de baseado no Modelo Conceitual de Horta. Foram avaliados 17 pacientes adultos no periodo pré-operatório de cirurgia cardíaca, sendo nove homens e oito mulheres, com idade média de 58 anos. Os diagnósticos de enfermagem identificados, segundo a Taxonomia I da NANDA, com freqüência superior a 50,0\% foram: Intolerância à atividade (88,2\%), Risco para infecção (76,5\%), Risco para disfunção neurovascular periférica (76,5\%), Déficit de conhecimento (76,5\%), Perfusão tissular cardiopulmonar alterada (70,6\%), Padrão respiratório ineficaz (70,6\%), Dor (70,6\%), Padrões de sexualidade alterados $(58,8 \%)$ e Distúrbio no padrão de sono $(52,9 \%)$.

Observou-se uma predominância dos diagnósticos relacionados às necessidades psicobiológicas.
\end{abstract}

\section{PALAVRAS-CHAVE \\ Diagnóstico de enfermagem. Cirurgia torácica. Cuidados pré-operatórios.}

\author{
ABSTRACT \\ The purpose of this study was to \\ identify nursing diagnosis of \\ patients in the preoperative \\ period of cardiac surgery. One \\ instrument was elaborated and \\ validated for data collection. \\ Such instrument was organized \\ according to Horta's Conceptual \\ Model. Seventeen adult patients \\ in the preoperative period of \\ cardiac surgery were evaluated, \\ of whom 09 were males and 08 \\ were females with the average of \\ 58 years old. After data \\ collection, the nursing diagnoses \\ were identified based on \\ NANDA's Taxonomy I. The \\ nursing diagnoses identified with \\ a higher frequency than 50\% \\ were: Activity Intolerance \\ (88.2\%), Infection risk (76.5\%), \\ Risk for peripheral neurovas- \\ cular dysfunction (76.5\%), \\ Knowledge deficit (on the \\ disease, surgery and anesthesia) \\ (76.5\%), Altered cardiopulmo- \\ nary tissue perfusion (70.6\%), \\ Ineffective airway pattern \\ (70.6\%); Pain (70.6\%), Altered \\ Sexual Patterns (58.8\%) and \\ Sleep Pattern Disturbance \\ (52.9\%). It was observed a \\ predominance of diagnoses \\ related to physiological needs.
}

\section{KEYWORDS}

Nursing diagnosis.

Thoracic surgery.

Preoperative care.

\section{RESUMEN}

El objetivo de este estudiofué identificar los diagnósticos de enfermería en pacientes de cirugía cardiaca en el periodo pre operatorio. Para la colecta de datos fué elaborado y validado un instrumento basado en el modelo conceptual de Horta (1979). Fueron evaluados 17 pacientes adultos de cirugía cardiaca en el periodo pre operatorio, de los cuales nueve hombres y ocho mujeres, con una edad media de 58 años. Los diagnósticos de enfermería, identificados según Taxonomia I de la NANDA con frecuencia superior a 50,0\% fueron: Intolerancia a la actividad (88,2\%), Riesgo a infección (76,5\%), Riesgo a disfunción neurovascular periférica (76,5\%), Déficit de conocimiento (76,5\%), Perfusión tisular cardiopulmonar alterada (70,6\%), Padrón respiratorio ineficaz (70,6\%), Dolor (70,6\%), Patrones de sexualidad alterados $(58,8 \%)$ y Disturbio en el patrón del sueño (52,9\%). Observamos predominio de los diagnósticos relacionados con las necesidades psicobiológicas.

\section{PALABRAS CLAVE}

Diagnóstico de enfermería. Cirugía torácica.

Cuidados preoperatorios.

\author{
* Estudo realizado com o \\ apoio da Fundação de \\ Amparo à Pesquisa do \\ Estado de São Paulo - \\ FAPESP. \\ 1 Mestre em Enfermagem \\ pela Escola de Enfer- \\ magem de Ribeirão \\ Preto da USP(EERP) \\ USP). Professora \\ Assistente da Facul- \\ dade de Enfermagem \\ do Hospital Israelita \\ Albert Einstein. \\ legaldeano@hotmail.com \\ 2 Professora Associada \\ do Departamento de \\ Enfermagem Geral \\ e Especializada da \\ EERP/USP \\ rizzardo@eerp.usp.br \\ 3 Enfermeira da Unidade \\ de Terapia Intensiva e \\ Unidade Coronariana \\ do Hospital das \\ Clínicas da Faculdade \\ de Medicina de \\ Ribeirão Preto - USP. \\ termutes@netsite.com.br
}




\section{INTRODUÇÃO}

$\mathrm{O}$ avanço da cardiologia gerou maior desenvolvimento e expansão dos cuidados de enfermagem a pacientes que se encontram no período perioperatório de cirurgia cardíaca. Os cuidados de enfermagem passaram a ser fundamentais para a recuperação do paciente submetido a cirurgia. Diante desse contexto, a enfermagem vem aprimorando seus conhecimentos e propondo novas alternativas de assistência, desenvolvendo uma metodologia própria de trabalho, fundamentada no método científico, isto é, fundamentada no processo de enfermagem.

O processo de enfermagem tem sido considerado uma possibilidade de resposta a sérios questionamentos referentes à qualidade do cuidado e ao desenvolvimento científico da profissão ${ }^{(1)}$.

Atualmente, o processo de enfermagem está descrito em cinco etapas: coleta de dados, diagnóstico, planejamento, implementação e avaliação ${ }^{(2)}$. Dentre essas etapas, o diagnóstico de enfermagem tem merecido grande destaque, sendo reconhecido como um guia para o planejamento e implementação dos cuidados de enfermagem ${ }^{(3)}$.

O diagnóstico de enfermagem representa uma das mais importantes fontes de conhecimento científico específico de enfermagem, fornecendo critérios mensuráveis para avaliação da assistência, além de direcionar o cuidado, facilitar a pesquisa e o ensino, estimular o cliente a participar de seu tratamento e do plano terapêutico e contribuir para expansão de um corpo de conhecimento próprio para a enfermagem ${ }^{(3)}$. Corresponde à segunda fase do processo de enfermagem, em que os dados coletados são analisados para o levantamento dos problemas de enfermagem. Esses problemas irão direcionar a assistência de enfermagem ${ }^{(4)}$.

Com o objetivo de classificar os diagnósticos de enfermagem, contribuir para uniformização da linguagem, fornecer dados para pesquisa em enfermagem e desenvolver um corpo de conhecimento próprio da profissão, a North American Nursing Association (NANDA) construiu uma estrutura conceitual de classificação denominada Taxonomia I dos Diagnósticos de Enfermagem ${ }^{(5)}$.
Os benefícios da implementação de ações direcionadas, fundamentadas e individualizadas, incentivaram-nos a identificar os diagnósticos de enfermagem de pacientes que se encontram no período pré-operatório de cirurgia cardíaca. A identificação dos diagnósticos de enfermagem de um grupo de clientes possibilita o conhecimento das respostas humanas alteradas, contribuindo assim para o desenvolvimento de intervenções de enfermagem direcionadas e individualizadas.

Vários autores têm se preocupado em identificar os diagnósticos de enfermagem em grupos específicos, como por exemplo, em pacientes hematológicos ${ }^{(6)}$, em pacientes queimados ${ }^{(7)}$, em pacientes com cardiopatia chagásica crônica ${ }^{(8)}$, em pacientes submetidos à terapia trombolítica ${ }^{(9)} \mathrm{e}$ em pacientes nos períodos pré e pós-operatórios de cirurgia cardíaca ${ }^{(10)}$. Logo, assim como este estudo, diversos trabalhos investigam os diagnósticos de enfermagem sem considerar, de imediato, as outras fases do processo ${ }^{(6,8,10-12)}$.

Mediante a identificação dos diagnósticos de enfermagem de pacientes no período pré-operatório de cirurgia cardíaca, pretende-se auxiliar os enfermeiros na elaboração de intervenções fundamentadas e adequadas às necessidades individuais de cada paciente, colaborando para a implementação de ações rápidas e eficazes para a resolução dos problemas identificados.

\section{OBJETIVO}

Identificar os diagnósticos de enfermagem de pacientes que se encontram no período pré-operatório de cirurgia cardíaca.

\section{CASUÍSTICA E MÉTODO}

Este estudo constitui parte de uma pesquisa intitulada Diagnósticos de enfermagem de pacientes no período perioperatório de cirurgia cardíaca, aprovada pelo Comitê de Ética e Pesquisa do Hospital das Clínicas da Faculdade de Medicina de Ribeirão Preto - USP e desenvolvida nesta mesma instituição.

O estudo foi realizado nas unidades de internação clínica e cirúrgica do Hospital das Clínicas da Faculdade de Medicina de Ri- 
beirão Preto, no período de janeiro a maio de 2001. O grupo de estudo foi constituído de 17 pacientes adultos, independente do sexo, que se encontravam no período pré-operatório de cirurgia cardíaca. Os critérios adotados para a inclusão no grupo foram: ser adulto; estar no período préoperatório de revascularização do miocárdio, troca ou plastia de valvas e demonstrar interesse na participação do estudo com assinatura do Termo de consentimento livre esclarecido.

Para a coleta de dados foi elaborado um instrumento, organizado de acordo as necessidades humanas básicas. Para a construção desse instrumento optou-se, dentre os diversos referenciais teóricos, pelo Modelo Conceitual de Wanda Horta ${ }^{(4)}$, por considerá-lo um modelo que permite a avaliação do paciente como um todo indivisível, com seus componentes biopsico-sócio-espirituais.

Seis enfermeiros, que atuavam na área de cardiologia e trabalhavam com diagnósticos de enfermagem, foram convidados a realizar a validação de forma e conteúdo do instrumento de coleta de dados. Para a validação foi elaborado um questionário, constituído de cinco questões, relacionadas à avaliação dos enfermeiros quanto à forma de apresentação e conteúdo do instrumento, e sugestões quanto à retirada, acréscimo ou modificações dos itens.

Realizada a validação dos instrumentos, iniciou-se um pré-teste mediante a aplicação do instrumento de coleta de dados em cinco pacientes, que atenderam aos critérios de inclusão, com os seguintes objetivos: identificar a melhor forma de aplicação dos instrumentos, verificar a adequação do conteúdo, identificar problemas que pudessem interferir na fidedignidade dos dados, avaliar o tempo gasto durante as entrevistas e promover os ajustes necessários.

Terminado o pré-teste e realizados os ajustes necessários, referentes à forma de apresentação do instrumento, foi iniciada a coleta de dados que procedeu da seguinte maneira: no dia anterior à cirurgia, após consultar a escala cirúrgica e a constatar de que o paciente era um provável integrante da amostra, a pesquisadora iniciava os esclarecimentos sobre o estudo e o indagava quanto ao desejo em participar do estudo. Mediante resposta positiva e consentimento por escrito, a pesquisadora iniciava as observações clínicas e a entrevista com o paciente.

Os dados coletados passaram pelos processos de análise e síntese e, após o processo de raciocínio diagnóstico ${ }^{(13)}$, foram estabelecidos os diagnósticos de enfermagem com base na Taxonomia I da $(\text { NANDA })^{(5)}$.

\section{RESULTADOS E DISCUSSÃO}

Foram avaliadas 17 pacientes, sendo 9 homens e 8 mulheres, com idade média de 58 anos.

O Quadro 1 indica as categorias diagnósticas identificadas segundo a Taxonomia I da NANDA no período pré-operatório de cirurgia cardíaca.

Observa-se no Quadro 1 que foram identificadas 33 categorias diagnósticas; dessas categorias, 9 apresentaram freqüência superior a $50,0 \%$.

Entre as categorias diagnósticas identificadas com freqüência superior a 50,0\% destacou-se a Intolerância à atividade, identificada em 15 pacientes $(88,2 \%)$. Dependendo do grau da insuficiência cardíaca os pacientes podem apresentar intolerância à atividade física e apresentar dificuldades para deambular, mesmo que por um curto período, devido ao desconforto respiratório, à fadiga e à palpitação que essa atividade pode acarretar. Além disso, o aparecimento de desconforto torácico durante o esforço físico faz, muitas vezes, com que o paciente limite sua atividade devido ao mal estar físico e ao medo de infarto de miocárdio ${ }^{(14)}$.

A categoria diagnóstica Risco para disfunção neurovascular periférica foi identificada em 14 pacientes, correspondendo a $82,4 \%$ do grupo. Destes pacientes, 12 possuíam diagnóstico médico de doença arterial coronariana e 2 possuíam diagnóstico de insuficiência mitral, fibrilação atrial crônica e história prévia de obstrução arterial aguda em membro inferior.
Diagnósticos de enfermagem de pacientes no período pré-operatório de cirurgia cardíaca 
Luzia Elaine Galdeano Lídia Aparecida Rossi Termutes Michelin Pezzuto

Quadro 1 - Categorias diagnósticas identificadas em indivíduos adultos no período pré-operatório de cirurgia cardíaca (Ribeirão Preto, janeiro a maio de 2001)

\begin{tabular}{|c|c|c|c|}
\hline & Categoria diagnóstica & $n^{0}$ & $\%$ \\
\hline 1) & Intolerância a atividade & 15 & $(88,2)$ \\
\hline 2) & Risco para disfunção neurovascular periférica & 14 & $(82,4)$ \\
\hline 3) & Risco para infecção & 13 & $(76,5)$ \\
\hline 4) & $\begin{array}{l}\text { Déficit de conhecimento (sobre a doença, a cirurgia e o período } \\
\text { pós-operatório)* }\end{array}$ & 13 & $(76,5)$ \\
\hline 5) & Padrão respiratório ineficaz & 12 & $(70,6)$ \\
\hline 6) & Perfusão tissular cardiopulmonar alterada & 12 & $(70,6)$ \\
\hline 7) & Dor & 12 & $(70,6)$ \\
\hline 8) & Padrões de sexualidade alterados & 10 & $(58,8)$ \\
\hline 9) & Distúrbio no padrão do sono & 9 & $(52,9)$ \\
\hline 10) & Ansiedade & 8 & $(47,1)$ \\
\hline 11) & Nutrição alterada: mais do que as necessidades corporais & 7 & $(41,2)$ \\
\hline 12) & Processos familiares alterados & 6 & $(35,3)$ \\
\hline 13) & Fadiga & 6 & $(35,3)$ \\
\hline 14) & Disfunção sexual & 3 & $(17,6)$ \\
\hline 15) & Constipação & 3 & $(17,6)$ \\
\hline 16) & Proteção alterada & 2 & $(11,8)$ \\
\hline 17) & Nutrição alterada: menos que as necessidades corporais & 2 & $(11,8)$ \\
\hline 18) & Mobilidade física prejudicada & 2 & $(11,8)$ \\
\hline 19) & Risco para lesão perioperatória de posicionamento & 2 & $(11,8)$ \\
\hline 20) & Integridade da pele prejudicada & 2 & $(11,8)$ \\
\hline 21) & Baixa auto-estima situacional & 2 & $(11,8)$ \\
\hline 22) & Eliminação urinária alterada & 1 & $(5,9)$ \\
\hline 23) & Troca de gases prejudicada & 1 & $(5,9)$ \\
\hline 24) & Comunicação verbal prejudicada & 1 & $(5,9)$ \\
\hline 25) & Deglutição prejudicada & 1 & $(5,9)$ \\
\hline 26) & Alteração sensorial visual/de perceção & 1 & $(5,9)$ \\
\hline 27) & Alteração sensorial auditiva/de percepção & 1 & $(5,9)$ \\
\hline 28) & Alteração sensorial tátil/de percepção & 1 & $(5,9)$ \\
\hline 29) & Déficit no autocuidado para banho/higiene & 1 & $(5,9)$ \\
\hline 30) & Risco para trauma & 1 & $(5,9)$ \\
\hline 31) & Risco para constipação & 1 & $(5,9)$ \\
\hline 32) & Manutenção de saúde alterada & 1 & $(5,9)$ \\
\hline 33) & Angústia espiritual & 1 & $(5,9)$ \\
\hline
\end{tabular}

* A informação assinalada representa especificidades apresentadas pelo paciente

Segundo a literatura, pacientes com doença arterial coronariana são portadores de aterosclerose e podem apresentar obstrução vascular em outros locais. Além disso, pacientes com fibrilação atrial podem apresentar trombos, formados dentro da câmara cardíaca, devido à estase do sangue, que podem se desprender do seu local de origem, migrar pelo sistema circulatório e levar a uma obstrução arterial aguda ${ }^{(15)}$.

O Risco para infecção foi identificado em 13 pacientes $(76,5 \%)$ que possuíam mais de 50 anos. Estudos apontam que pacientes com menos de um ano e mais de 50 anos possuem maiores taxas de infecção ${ }^{(17)}$.

É importante ressaltar que dos pacientes que apresentaram Risco para infecção, $7(41,2 \%)$ eram obesos e, portan- to, possuíam Nutrição alterada: mais do que as necessidades corporais (Quadro 1). A obesidade é um fator de risco comprovado para infecções, principalmente nos períodos trans e pósoperatórios. Estudos comprovam que a espessura do tecido gorduroso tem influência direta e proporcional nas taxas de infecção. As razões para essa susceptibilidade estão relacionadas à irrigação local do tecido adiposo, pouco vascularizado, e à maior duração dos procedimentos cirúrgicos ${ }^{(16-17)}$.

Ainda de acordo com o Quadro 1, observa-se que 13 pacientes $(76,5 \%)$ possuíam Déficit de conhecimento relacionado à doença, cirurgia, anestesia e período pósoperatório. Sabe-se que o ensino dos pacientes tem influência direta sobre as fases posteriores a cirurgia, levando-os ao desenvolvimento de sua independência 
em relação à equipe de saúde e à redução dos sentimentos de medo e insegurança ${ }^{(18)}$. Diante disso torna-se fundamental ajudar os pacientes, no período pré-operatório, a compreenderem questões relacionadas ao seu estado de saúde e à cirurgia, com o objetivo de ajudá-los a enfrentar a hospitalização e a cirurgia e tornar essas situações menos ameaçadoras e conflitantes.

A categoria diagnóstica Perfusão tissular cardiopulmonar alterada foi identificada em 12 pacientes $(70,6 \%)$ que possuíam diagnóstico médico de doença arterial coronariana. Nas coronariopatias, o calibre das coronárias se encontra reduzido devido a lesões ateroscleróticas, diminuindo o suprimento sanguíneo para determinada área do coração. Essa diminuição do aporte sanguíneo e a conseqüente redução do suprimento de oxigênio afetam o metabolismo das células cardíacas podendo alterar suas funções contráteis e elétricas ${ }^{(19)}$.

O Padrão respiratório ineficaz foi identificado em 12 pacientes $(70,6 \%)$ que referiam uma sensação de "fome de ar" ou de sentir "maior necessidade de respirar", principalmente ao fazer esforço. Esse resultado é esperado já que sabe-se que a dispnéia, a respiração desconfortável, difícil e trabalhosa, é freqüentemente observada em pacientes com doença cardíaca, e se agrava com a atividade física (dispnéia aos esforços) $)^{(15)}$.

A categoria diagnóstica Dor foi identificada em 12 pacientes $(70,6 \%)$, que a descreveram como uma sensação de constrição, aperto ou queimação na região esternal, irradiando-se com freqüência, para o ombro esquerdo, para o pescoço ou para o braço. A dor cardíaca é ocasionada pela constrição progressiva das artérias coronárias, reduzindo o fluxo coronariano. É comumente desencadeada por qualquer fator que resulte no aumento da demanda miocárdica de oxigênio, como o esforço ou emoção, e é aliviada pelo repouso. Acredita-se que a isquemia faça o músculo cardíaco liberar substâncias ácidas, como o ácido lático, histamina, cininas ou enzimas proteolíticas celulares que, quando em elevadas concentrações, estimulam as terminações nervosas do músculo cardíaco produzindo a dor ${ }^{(20)}$.

Destaca-se também a categoria diagnóstica Padrões de sexualidade alterados identificada em 10 pacientes $(58,8 \%)$. Como os demais aspectos da vida, a sexualidade sofre interferência do adoecer e do tratamento. O estabelecimento dessa categoria se deu mediante relatos de preocupação com o "desempenho sexual" e de mudança em relação à atividade sexual, devido à intolerância a esforços, como "falta de ar", "palpitação", ou "dor no peito". Muitas são as causas de disfunção sexual em pacientes com problemas cardíacos, entre elas podemos citar os fatores psicológicos, as doenças associadas e aos efeitos dos medicamentos utilizados por esse grupo de pacientes. O medo de complicações cardíacas durante o ato sexual é responsável por grande parte dessa disfunção ${ }^{(14)}$.

O Distúrbio no padrão de sono foi identificado em 9 pacientes $(52,9 \%)$. A impossibilidade de dormir e de sentir-se repousado é uma queixa comum durante a hospitalização, tendo como principais causas a falta de "intimidade" com o ambiente, a dor, o desconforto, a dependência e as sensações de perda do autocontrole ${ }^{(21)}$.

Estão apresentados no Quadro 2, os fatores relacionados às categorias diagnósticas identificadas no período préoperatório, cuja freqüência foi superior a $50 \%$.

O Quadro 2 indica que para a categoria diagnóstica de maior freqüência Intolerância a atividade (88,2\%) foi identificada apenas um fator relacionado, o desequilibrio entre a oferta e a demanda de oxigênio (100,0\%). O mesmo ocorreu para as categorias: Déficit de conhecimento com o fator relacionado falta de exposição (100,0\%); Dor, com agentes lesivos biológicos (100,0\%) e Perfusão tissular cardiopulmonar alterada, com redução mecânica do fluxo sangüíneo arterial (doença arterial coronariana) $(100,0 \%)$.
Diagnósticos de enfermagem de pacientes no período pré-operatório de cirurgia cardíaca 
Luzia Elaine Galdeano Lídia Aparecida Rossi Termutes Michelin Pezzuto
Quadro 2 - Fatores relacionados às categorias diagnósticas identificadas em indivíduos adultos no período pré-operatório de cirurgia cardíaca (Ribeirão Preto, janeiro a maio de 2001)

\begin{tabular}{|c|c|c|c|}
\hline Categoria diagnóstica & Fatores relacionados & $n^{0}$ & $\%$ \\
\hline Intolerância a atividade $(n=15)$ & - Desequilíbrio entre a oferta e a demanda de oxigênio & 15 & $(100,0)$ \\
\hline Déficit de conhecimento $(n=13)$ & $\begin{array}{l}\text { - Falta de exposição (de informações relacionadas } \\
\text { ao procedimento anestésico-cirúrgico) }\end{array}$ & 13 & $(100,0)$ \\
\hline Padrão respiratório ineficaz $(n=12)$ & $\begin{array}{l}\text { - Energia diminuída/fadiga } \\
\text { - Posição do corpo (decúbito dorsal)* } \\
\text { - Obesidade }\end{array}$ & $\begin{array}{l}12 \\
8 \\
4\end{array}$ & $\begin{array}{r}(100,0) \\
(66,6) \\
(33,3)\end{array}$ \\
\hline $\begin{array}{l}\text { Perfusão tissular cardiopulmonar } \\
\text { alterada }(n=12)\end{array}$ & $\begin{array}{l}\text { - Redução mecânica do fluxo sangüíneo arterial } \\
\text { (doença arterial coronariana)* }\end{array}$ & 12 & $(100,0)$ \\
\hline Dor $(n=12)$ & - Agentes lesivos biológicos & 12 & $(100,0)$ \\
\hline $\begin{array}{l}\text { Padrões de sexualidade alterados } \\
(n=10)\end{array}$ & $\begin{array}{l}\text { - Doença } \\
\text { - Relacionamento prejudicado com pessoa significativa }\end{array}$ & $\begin{array}{l}9 \\
1\end{array}$ & $\begin{array}{l}(90,0) \\
(10,0)\end{array}$ \\
\hline $\begin{array}{l}\text { Distúrbio no padrão do sono } \\
(n=10)\end{array}$ & $\begin{array}{l}\text { - Urgência urinária } \\
\text { - Falta de ar } \\
\text { - Posição } \\
\text { - Esquema de dormir e acordar que muda freqüentemente } \\
\text { - Barulho e iluminação } \\
\text { - Despertar causado por outros (equipe de enfermagem)* } \\
\text { - Medo } \\
\text { - Ansiedade } \\
\text { - Pensamentos ruminantes pré-sono } \\
\text { - Separação de pessoas significativas (filhos e netos) }\end{array}$ & $\begin{array}{l}6 \\
4 \\
4 \\
4 \\
3 \\
3 \\
2 \\
2 \\
2 \\
1\end{array}$ & $\begin{array}{l}(66,6) \\
(44,4) \\
(44,4) \\
(44,4) \\
(33,3) \\
(33,3) \\
(22,2) \\
(22,2) \\
(22,2) \\
(11,1)\end{array}$ \\
\hline
\end{tabular}

* As informações assinaladas representam especificidades apresentadas pelos pacientes

A categoria diagnóstica que apresentou maior número de fatores relacionados foi o Distúrbio no padrão do sono. Para esta categoria foram identificados 10 fatores relacionados diferentes, sendo o de maior freqüência a urgência urinária, identificada em $66,6 \%$ dos pacientes. Nesse caso, a urgência urinária pode ser justificada pelo fato de pacientes com insuficiência cardíaca congestiva apresentarem nictúria, secundária à absorção do edema durante a noite ${ }^{(19)}$. Uma outra justificativa é a ansiedade, apresentada como fator relacionado a esse diagnóstico em dois pacientes (22,2\%). É importante ressaltar que a NANDA ${ }^{(5)}$ apresenta a urgência urinária como uma manifestação fisiológica do diagnóstico de ansiedade.

Estão apresentadas, no Quadro 3, as características definidoras das categorias diagnósticas identificadas no período pré-operatório, cuja freqüência foi superior a $50 \%$.

No Quadro 3 observa-se que as categorias diagnósticas Déficit de conhecimento e Dor apresentaram apenas uma característica definidora, que foram respectivamente verbalização (de não ter conhecimento sobre a doença, tratamento ou período pós-operatório) $(100,0 \%)$ e relato verbal (de sentir dor) $(100,0 \%)$.
A categoria diagnóstica Padrão respiratório ineficaz $(70,6 \%)$ foi caracterizada com maior freqüência por dispnéia $(100,0 \%)$ e a Perfusão tissular cardiopulmonar alterada por dor no peito $(91,6 \%)$ e dispnéia $(83,3 \%)$.

De acordo com o Quadro 3, a insatisfação com o sono foi identificada em 8 pacientes (88,8\%); esta manifestação do Distúrbio do padrão do sono, está diretamente relacionada as outras características definidoras como: três ou mais despertares durante a noite identificada em 7 pacientes (77,7\%) em conseqüência da ortopnéia e nictúria apresentadas por esses pacientes e queixas verbais de dificuldade para adormecer, também identificada em 7 pacientes $(77,7 \%)$.

A característica definidora mais freqüente para a categoria diagnóstica Padrões de sexualidade alterados foi mudanças relatadas nos comportamentos ou atividades sexuais (90,0\%), normalmente devido à Intolerância à atividade, manifestada por: desconforto ou dispnéia de esforço (93,3\%), por relato verbal de fraqueza ou fadiga $(80,0 \%)$ e por resposta anormal da freqüência cardíaca ou da pressão sanguínea à atividade $(46,6 \%)$.
Rev Esc Enferm USP 2004; 38(3): 307-16. 
Quadro 3 - Características definidoras das categorias diagnósticas identificadas em indivíduos adultos no período pré-operatório de cirurgia cardíaca (Ribeirão Preto, janeiro a maio de 2001)

Diagnósticos de enfermagem de pacientes no período pré-operatório de cirurgia cardíaca

\begin{tabular}{|c|c|c|c|}
\hline Categoria diagnóstica & Características definidoras & $n^{0}$ & $\%$ \\
\hline Intolerância a atividade $(n=15)$ & $\begin{array}{l}\text { - Desconforto ou dispnéia de esforço } \\
\text { - relato verbal de fraqueza ou fadiga } \\
\text { - Resposta anormal da freqüência cardíaca ou da pressão } \\
\quad \text { sangüínea à atividade }\end{array}$ & $\begin{array}{c}14 \\
12 \\
7\end{array}$ & $\begin{array}{l}(93,3) \\
(80,0) \\
(46,6)\end{array}$ \\
\hline Déficit de conhecimento $(n=13)$ & $\begin{array}{l}\text { - Verbalização (de não ter conhecimento sobre a doença, } \\
\text { tratamento ou período pós-operatório) }\end{array}$ & 13 & $(100,0)$ \\
\hline Padrão respiratório ineficaz $(n=12)$ & $\begin{array}{l}\text { - Dispnéia } \\
\text { - Ortopnéia } \\
\text { - Respiração curta }\end{array}$ & $\begin{array}{l}12 \\
8 \\
2\end{array}$ & $\begin{array}{r}(100,0) \\
(66,6) \\
(16,6)\end{array}$ \\
\hline $\begin{array}{l}\text { Perfusão tissular cardiopulmonar } \\
\text { alterada }(n=12)\end{array}$ & $\begin{array}{l}\text { - Dor no peito } \\
\text { - Dispnéia } \\
\text { - Freqüência respiratória aumentada, fora dos } \\
\quad \text { parâmetros aceitáveis }\end{array}$ & $\begin{array}{c}11 \\
10 \\
1\end{array}$ & $\begin{array}{r}(91,6) \\
(83,3) \\
(8,3)\end{array}$ \\
\hline Dor $(n=12)$ & - Relato verbal (da dor) & 12 & $(100,0)$ \\
\hline $\begin{array}{l}\text { Padrões de sexualidade alterados } \\
(n=10)\end{array}$ & $\begin{array}{l}\text { - Mudanças relatadas nos comportamentos } \\
\text { ou atividades sexuais } \\
\text { - Dificuldades, limitações e mudanças } \\
\text { nas atividades sexuais }\end{array}$ & 9 & $\begin{array}{l}(90,0) \\
(40,0)\end{array}$ \\
\hline $\begin{array}{l}\text { Distúrbio no padrão do sono } \\
(n=10)\end{array}$ & $\begin{array}{l}\text { - Insatisfação com o sono } \\
\text { - Três ou mais despertares durante a noite } \\
\text { - Queixas verbais de dificuldade para adormecer } \\
\text { - Insônia de manutenção de sono } \\
\text { - Despertar mais cedo do que o desejado } \\
\text { - Queixas verbais de não se sentir bem descansado }\end{array}$ & $\begin{array}{l}8 \\
7 \\
7 \\
3 \\
1 \\
1\end{array}$ & $\begin{array}{l}(88,8) \\
(77,7) \\
(77,7) \\
(33,3) \\
(11,1) \\
(11,1)\end{array}$ \\
\hline
\end{tabular}

O Quadro 4 indica os fatores de risco das categorias diagnósticas de risco identi- ficadas, no período pré-operatório de cirurgia cardíaca, cuja freqüência foi superior a $50 \%$.

Quadro 4 - Fatores de risco das categorias diagnósticas identificadas em indivíduos adultos no período pré-operatório de cirurgia cardíaca (Ribeirão Preto, janeiro a maio de 2001)

\begin{tabular}{|llcc|}
\hline \multicolumn{1}{|c}{ Categoria diagnóstica } & \multicolumn{1}{c|}{ Fatores de risco } & $\mathbf{n}^{\mathbf{0}}$ & $\%$ \\
\hline $\begin{array}{l}\text { Risco para disfunção } \\
\text { neurovascular periférica }(\mathrm{n}=14)\end{array}$ & $\begin{array}{l}\text { - Obstrução vascular } \\
\text { (doença arterial coronariana) }\end{array}$ & 14 & $(100,0)$ \\
& & & \\
Risco para infecção $(\mathrm{n}=13)$ & - Exposição ambiental aumentada & 10 & $(76,9)$ \\
& - Doença crônica (Diabetes Melitos) & \\
& - Defesa primária inadequada (pele rompida, & 2 & $(15,4)$ \\
& - tecido traumatizado) & 7 & \\
& - Procedimentos invasivos (punção venosa) & 1 & $(7,7)$ \\
\hline
\end{tabular}

* As informações assinaladas representam especificidades apresentadas pelos pacientes

De acordo com o Quadro 4, o fator de risco identificado com maior freqüência para a categoria diagnóstica Risco para infecção foi a exposição ambiental aumentada, identificada em 10 pacientes (76,9\%). O termo "exposição ambiental aumentada" possibilita uma ampla margem de entendimento e gera dúvidas. Inúmeras vezes foi questionado so- bre quais seriam os fatores que poderiam levar o paciente a uma "exposição ambiental aumentada". Com embasamento na literatura, padronizou-se que o fato do paciente estar internado há mais de dois dias, independente de ter sido submetido a procedimentos invasivos, seria suficiente para definir a exposição ambiental aumentada. Para
Rev Esc Enferm USP 2004; 38(3):307-16. 
Luzia Elaine Galdeano Lídia Aparecida Rossi Termutes Michelin Pezzuto isso, foram considerados alguns estudos sobre o assunto que se reportam aquisição da flora hospitalar pelo paciente e demonstram que a colonização da pele por microrganismos patogênicos aumenta proporcionalmente ao tempo de hospitalização ${ }^{(16-17)}$.

Entre os fatores de risco identificados com maior freqüência neste estudo, está a doença crônica (53,8\%), estabelecida em pacientes com Diabetes Melitos. A presença de diabetes é um importante fator de risco para infecção, devido às alterações na cicatrização, às complicações vasculares e neuropáticas e aos defeitos inibitórios nos mecanismos de defesa ${ }^{(16)}$.

Avaliando o Quadro 5, pode-se observar uma predominância dos diagnósticos relacionados às necessidades psicobiológicas. Observa-se que dos 33 diagnósticos de enfermagem identificados (Quadro 1), 26 (78,8\%) estão relacionados às necessidades psicobiológicas, $6(18,2 \%)$ estão relacionados às necessidades psicossociais e apenas $1(3 \%)$ está relacionado às necessidades psicoespirituais.

Quadro 5 - Categorias diagnósticas identificadas em indivíduos adultos no período pré-operatório de cirurgia cardíaca (Ribeirão Preto, janeiro a maio de 2001)

\begin{tabular}{|c|c|}
\hline \multicolumn{2}{|c|}{ Necessidades psicobiológicas } \\
\hline $\begin{array}{l}\text { - Oxigenação } \\
\text { Padrão respiratório ineficaz (5) } \\
\text { Troca de gases prejudicada (23) } \\
\text { - Nutrição } \\
\text { Nutrição alterada: mais do que as necessidades corporais (11) } \\
\text { Nutricão alterada: menos do que as necessidades corporais (12) } \\
\text { Deglutição prejudicada (25) } \\
\text { - Eliminação } \\
\text { Constipação (15) } \\
\text { Risco para constipação (31) } \\
\text { Eliminação urinária alterada (22) } \\
\text { - Sono e repouso } \\
\text { Distúrbio no padrão do sono (9) } \\
\text { - Exercício e atividade física } \\
\text { Intolerância a atividade (1) } \\
\text { Padrões de sexualidade alterados (8) } \\
\text { Mobilidade física prejudicada (18) } \\
\text { - Sexualidade } \\
\text { (isfuno sexual }\end{array}$ & $\begin{array}{l}\text { - Cuidado corporal } \\
\text { Déficit no autocuidado para banho/ higiene (29) } \\
\text { - Integridade cutâneo-mucosa } \\
\text { Integridade da pele prejudicada (20) } \\
\text { - Integridade física } \\
\text { Risco p/ lesão perioperatória de posicionamento (19) } \\
\text { Fadiga (13) } \\
\text { Risco para trauma (30) } \\
\text { Risco para infecção (3) } \\
\text { Proteção alterada (16) } \\
\text { - Regulação vascular } \\
\text { Risco para disfunção neurovascular periférica (2) } \\
\text { Perfusão tissular cardiopulmonar alterada (6) } \\
\text { - Percepção } \\
\text { Dor (7) } \\
\text { Alteração sensorial visual de percepção (26) } \\
\text { Alteração sensorial auditiva de percepção (27) } \\
\text { Alteração sensorial tátil de percepção (28) }\end{array}$ \\
\hline \multicolumn{2}{|c|}{ Necessidades psicosociais } \\
\hline $\begin{array}{l}\text { - Segurança } \\
\text { Ansiedade (10) } \\
\text { - Amor/ Gregária } \\
\text { Processos familiares alterados (12) } \\
\text { - Comunicação } \\
\text { Comunicação verbal prejudicada (24) }\end{array}$ & $\begin{array}{l}\text { - Aprendizagem } \\
\text { Déficit de conhecimento (4) } \\
\text { - Orientação } \\
\text { Manutenção de saúde alterada (32) } \\
\text { - Auto-estima } \\
\text { Baixa auto-estima situacional (21) }\end{array}$ \\
\hline \multicolumn{2}{|c|}{ Necessidades psicoespirituais } \\
\hline $\begin{array}{l}\text { - religiosa ou teológica, ética ou de filosofia de vida } \\
\text { Angústia espiritual (33) }\end{array}$ & \\
\hline
\end{tabular}


A predominância dos diagnósticos relacionados às necessidades psicobiológicas é esperada e justificada pela hospitalização e pelo processo da doença cardíaca. $\mathrm{Na}$ abordagem adotada neste estudo ${ }^{(4)}$, as necessidades físiológicas ou de sobrevivência são as de maior prioridade, pois são as que, em geral, os indivíduos procuram satisfazer em primeiro lugar.

\section{CONCLUSÕES}

Mediante os resultados identificados neste estudo conclui-se que:

- as categorias diagnósticas identificadas, segundo a Taxonomia I da NANDA, com freqüência superior a $50 \%$ foram: Intolerância à atividade $(88,2 \%)$, Risco para disfunção neurovascular periférica $(82,4 \%)$, Risco para infecção (76,5\%), Déficit de conhecimento (76,5\%), Perfusão tissular cardiopulmonar alterada (70,6\%), Padrão respiratório ineficaz (70,6\%), Dor (70,6), Padrões de sexualidade alterados $(58,8 \%)$ e Distúrbio no padrão do sono $(52,9 \%)$;

- das 33 categorias diagnósticas identificadas, $26(78,8 \%)$ estão relacionadas às necessidades psicobiológicas, 6 (18,2\%) estão relacionadas às necessidades psicossociais e $1(3 \%)$ está relacionada às necessidades psicoespirituais; observamos também que 28 (84,8\%) são categorias diagnósticas reais e $5(15,2 \%)$ são categorias diagnósticas de risco.

\section{CONSIDERAÇÕES FINAIS}

Apesar da predominância dos diagnósticos de enfermagem relacionados à área psicobiológica, justificada pelo processo saúde-doença que envolve diversos mecanismos fisiológicos, não deve-se deixar de investigar as necessidades psicossociais e o impacto psicológico da doença cardíaca, responsável por grandes mudanças na qualidade de vida do paciente e sua família e que possuem um papel importante na capacidade do indivíduo de suprir suas outras necessidades básicas ${ }^{(4)}$.

Os resultados obtidos neste estudo favorecem o direcionamento da assistência com base no processo de enfermagem, subsidiam estudos similares e conduzem os profissionais na elaboração de um plano de cuidados individualizado, levando ao fortalecimento da atenção a pacientes que se encontram no período pré-operatório de cirurgia cardíaca e a melhora na qualidade da assistência de enfermagem prestada.

O conhecimento dos problemas de saúde de um grupo de pacientes com características comuns, irá direcionar a assistência de enfermagem, fornecendo subsídios para a elaboração do plano de cuidados, implementação de intervenções, treinamento e qualificação da equipe de enfermagem. O presente estudo contribuirá para o incremento de pesquisas sobre o processo de enfermagem e, conseqüentemente, para o desenvolvimento técnico-científico da profissão.

Considerando importância da implantação de uma assistência integral e individualizada, voltada às necessidades do paciente durante todas as fases do seu tratamento, é fundamental a realização de estudos voltados à identificação dos diagnósticos de enfermagem em todo o período perioperatório. A assistência de enfermagem perioperatória deve ser individualizada, contínua e planejada, compreendendo o paciente em todas as fases, mediante a avaliação de enfermagem nos períodos pré, trans e pós-operatórios ${ }^{(22)}$.
Diagnósticos de enfermagem de pacientes no período pré-operatório de cirurgia cardíaca

\section{REFERÊNCIAS}

(1) Pimenta CAM, Cruz DALM, Araújo TL, Graziano KU, Kimura M, Miyadahira AMK. O ensino da avaliação do paciente: delineamento do conteúdo pelo diagnóstico de enfermagem. Rev Lat Am Enferm 1993; 1(2):69-76.

(2) Potter PA, Perry AG. Grande tratado de enfermagem prática - conceitos básicos, teoria e prática hospitalar $2^{\mathrm{a}}$ ed. São Paulo: Santos; 1997.
(3) Jesus CAC. Raciocínio clínico de graduandos e enfermeiros na construção de diagnósticos de enfermagem. [tese] Ribeirão Preto (SP): Escola de Enfermagem de Ribeirão Preto/USP; 2000.

(4) Horta WA. Processo de enfermagem. São Paulo: EDUSP; 1979.

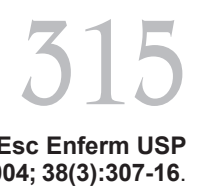


Luzia Elaine Galdeano Lídia Aparecida Rossi Termutes Michelin Pezzuto
(5) North American Nursing Diagnosis Association - NANDA. Diagnósticos de enfermagem: definições e classificações. Porto Alegre: Artes Médicas; 1999-2000.

(6) Jesus CAC. Assistência de enfermagem a clientes hematológicos: uma visão sistêmica. [dissertação] Ribeirão Preto (SP): Escola de Enfermagem de Ribeirão Preto/ USP; 1992.

(7) Dalri MCB. Perfil diagnóstico de pacientes queimados segundo modelo conceitual de Horta e a Taxonomia I da NANDA. [dissertação] Ribeirão Preto (SP): Escola de Enfermagem de Ribeirão Preto/USP; 1993.

(8) Cruz DALM. Diagnósticos de enfermagem e padrões funcionais de saúde alterados em pacientes internados por cardiopatia chagásica crônica. [dissertação] São Paulo (SP): Escola de Enfermagem da USP; 1989.

(9) Riccio GMG, Augusto RR, Teixeira FFT, Silva CA. Diagnóstico de enfermagem dos pacientes submetidos à terapêutica trombolítica. Rev Soc Cardiol Estado São Paulo $1994 ; 4(3$ supl A):6-16.

(10) Faria MFG. Diagnósticos de enfermagem respiratórios em pacientes cardíacos cirúrgicos. [dissertação] São Paulo (SP): Escola de Enfermagem da USP; 2000.

(11) Carvalho EC, Jesus CAC. Raciocínio clínico na formulação do diagnóstico de enfermagem para o indivíduo. In: Antunes MJ, Silva MTN, organizadores. O uso do diagnóstico na prática de enfermagem. Brasília: ABEn; 1997. p. 27-38.

(12) Lopes RAM, Macedo DD, Lopes MHBM. Diagnósticos de enfermagem mais freqüentes em uma unidade de internação oncológica. Rev Lat Am Enferm 1997; 5(4):35-41.
(13) Risner PB. Diagnosis: analysis and synthesis of data. In: Christensen PJ, Kenney JW. Nursing process: conceptual models. $4^{\text {th }}$ ed. St. Louis: Mosby; 1990. p. 132-57.

(14) Maiello JR, Megale PLD, Nakazone S, Santos Júnior W. Retorno ao trabalho e qualidade de vida pós-infarto do miocárdio. Rev Soc Cardiol Estado São Paulo 1995; 5: 94-9.

(15) Goldman L, Braunwald EB. Cardiologia na clínica geral. Rio de Janeiro: Guanabara Koogan; 2000.

(16) Rabhae GN, Ribeiro Filho N, Fernandes AT. Infecção do sítio cirúrgico. In: Fernandes AT, editor. Infecção hospitalar e suas interfaces na área de saúde. São Paulo: Atheneu; 2000. p. 479-502.

(17) Huchcroft AS, Nicolle LE, Cruse PJ. Surgical wound infection and cancer among elderey: a case control study. J Surg Oncol 1990; 45(4):250-60.

(18) Zago MMF. Considerações sobre o ensino do paciente cirúrgico. Rev Esc Enferm USP 1993; 27(1):67-71.

(19) Andreoli TE, Bennett JC, Carpenter CCJ. Cecil: medicina interna básica. $3^{\mathrm{a}}$ ed. Rio de Janeiro: Guanabara Koogan; 1994.

(20) Guyton, AC. Tratado de fisiologia médica. $8^{\mathrm{a}}$ ed. Rio de Janeiro: Guanabara Koogan; 1992.

(21) Atkinson LD, Murray ME. Fundamentos de enfermagem: introdução ao processo de enfermagem. Rio de Janeiro: Guanabara Koogan; 1989.

(22) Castellanos BEP, Jouclas VMG. Assistência de enfermagem perioperatória: um modelo conceitual. Rev Esc Enferm USP 1990; 24(3):359-70 